\title{
Dignostic Utility of HBME1 to Differentiate Between Reactive Mesothelial Cells and Adenocarcinoma Cells in Body Fluids
}

\author{
Charusheela Gore, Karanpatel, Rupali Bavikar* and Ashily \\ Dept. of Pathology, Dr. D. Y. Patil Medical College, Hospital \& Research Centre \& DPU, Pune, Maharashtra
} \begin{abstract}
the benign cases showed membranous potivity.
\section{Introduction}

Serous cavities in the body are lined by mesothelial cells .Any pathology like infection, trauma, or neoplasia in serous cavities leads to hypertrophy and proliferation of mesothelial cells. These reactive mesothelial cells show variable morphological spectrum . They may show features overlapping with malignant cells often leading to false positive reports of serous fluids.
\end{abstract}

ABSTRACT

Background: Reactive mesothelial cells show overlapping features with malignant cells. To differentiate between the two Immunocytochemistry (ICC) can be used.HBME1 is the antigen present on mesothelial cells. We have tried to evaluate the efficacy of HBME1 to differentiate between reactive mesothelial cells and adenocarcinoma cells.

Methods: Total 60 cases with clinical dilemma or suspicious on conventional cytology were selected .Cell block was prepared and staining with monoclonal antibody HBME1 was done.Staining pattern was classified as cytoplasmic ,membranous or combined.

Result: Out of 42,28 cases showed negative staining , 8 cases showed cytoplasmic positivity and 4 cases showed combined positivity.All

Conclusion: The staining pattern of HBME1 can be used to differentiate between reactive mesothelial cells and adenocarcinoma cells.
Immunocytochemstry (ICC) is preferred method to differentiate between reactive mesothelial cells and adenocarcinoma. ${ }^{[1,2]}$ ICC done on cell block and cytospin is cost effective and shows better results as compared to flow cytometry. ${ }^{[3,4]}$ There are many mesothelial markers including Calretinin ,thrombomodulin,cytokratin 5/6, and HMBE $1 .{ }^{[5,6]}$ HMBE 1 is monoclonal antibody which reacts with surface antigen on mesothelial cells. ${ }^{[5,6]}$

This study is done to evaluate the diagnostic utility of HBME1 to distinguish between mesothelial cells from adenocarcinoma.

\section{Material and Methods}

This is prospective study from July 2010 to June 2012 done in department of pathology in DY Patil Medical college Pune.Total sixty cases of pleural or peritoneal effusions were selected for study.

Inclusion criteria was ,cases with diagnostic dilemma clinically or on conventional cytology, malignant pleural effusions, and few benign pleural effusions were also included. The diagnosis of each case was confirmed by computer tomography, bronchoscopy, surgical exicision and histhopathological examination.

The specimen (pleural or peritoneal fluid) was cytocentrifuged at $800 \mathrm{rpm}$ for 3 minuts. The cell button was taken on labeled and paraffin coated slides .The slides were immunostained using two step polymer (ENVISION ${ }^{\mathrm{TM}}$ ) method. Primary antibody used was Monoclonal anti mesothelial antibody clone HBME 1 (Dako) .The DAB (3,3 Diaamininobenzine) was used as chromogen and nuclei were counter stained with Mayer's Haematoxylene. The staining pattern was classified as membranous(thin and thick), cytoplasmic and combined (membranous +cytoplasmic).

\section{Results}

Out of 60 cases $50 \%$ were males and $50 \%$ were females. The incidence of malignant effusion was higher $(80 \%)$ in females as compared to males $(60 \%)$ ). Total 42 cases were malignant effusions .In malignant effusions ,36 $(85.8 \%)$ cases were that adenocarcinoma followed by 2 cases sqaumous cell carcinoma and 2 cases of Ewing's sarcoma (Table 1).In adenocarcinoma 16 out of 36 were from alimentary tract followed by lung(8) and ovary. (8). (Table 2)

The staining pattern of HBME1 in benign cases was membranous in 12 cases and combined in two cases (table3)(Figure1) .Out of 42 malignant cases, 28 cases 
showed negative staining for HBME1(Figure 2) ,8 cases showed cytoplasmic positivity(Figure 3),4 cases showed combined(Figure4 ) and only2 cases showed membranous positivity.(table 4)

Staining pattern in adenocarcinoma was cytoplasmic and combined except ovarian malignancy .2 cases of ovarian malignancy showed membranous positivity.(Table V)

\section{Discussion}

The reactive mesothelial cells may show changes like nucleomegaly,irregular nuclear membrane, coarse chromatin and conspicuous nucleoli, difficult to differentiate from malignant cells. The reactive mesothelial cells, with degenerative intracytoplasmic vacuoles may be misinterpreted as adenocarcinoma cells with mucin

Table I : Subtyping of Malignant Lesions

\begin{tabular}{|c|c|}
\hline Subtype of malignant lesions & No. of Cases \\
\hline Adenocarcinoma & 36 \\
\hline Squamous cell carcinoma & 2 \\
\hline Ewings sarcoma & 2 \\
\hline Signet ring lymphoma & 1 \\
\hline Non-Hodgkin's Lymphoma & 1 \\
\hline
\end{tabular}

Table II : Classification of Adenocarcinoma By Specific Origin $(\mathrm{N}=36)$

Type of adenocarcinoma by specific origin

\begin{tabular}{|c|c|}
\hline Ovary & 8 \\
\hline Lung & 8 \\
\hline Breast & 4 \\
\hline Alimentary Tract & 16 \\
\hline
\end{tabular}

Table III: HBME-1 Staining in Benign or Reactive Lesions.

\begin{tabular}{|c|c|c|c|c|}
\hline Negative & \multicolumn{3}{|c|}{ Positive } & Total No. of cases \\
\hline & Membranous & Cytoplasmic & Combined & \\
\hline 4 & 12 & 0 & 2 & 18 \\
\hline
\end{tabular}

Table IV: HBME-1 Staining in Malignant Lesions

\begin{tabular}{|c|c|c|c|c|}
\hline Negative & \multicolumn{3}{|c|}{ Positive } & Total No. of cases \\
\hline & Membranous & Cytoplasmic & Combined & \\
\hline 28 & 2 & 8 & 4 & 42 \\
\hline
\end{tabular}

Table V - Staining pattern in specific type of adenocarcinoma

\begin{tabular}{|c|c|c|c|c|c|}
\hline & Negative & Membranous & Cytoplasmic & Combined & Total \\
\hline Ovary & 2 & 2 & 2 & 2 & 0 \\
\hline Lung & 6 & 0 & 0 & 0 & 4 \\
\hline Breast & 4 & 0 & 4 & 2 & 16 \\
\hline Alimentary canal & 10 & 0 & 2 & \\
\hline
\end{tabular}

TABLE VI -Predictive Value,Sensitivity and Specificity of HBME1 in differentiating reactive mesothelial cells and adenocarcinoma cells.

\begin{tabular}{|c|c|c|}
\hline & Calculated Value & 95\% C.I \\
\hline Positive Predictive Value(PPV) & $50 \%$ & $24 \%-76 \%$ \\
\hline Negative Predictive Value(NPV) & $87.5 \%$ & $60.4 \%-97.8 \%$ \\
\hline Sensitivity & $77.8 \%$ & $40.2 \%-96.1 \%$ \\
\hline Specificity & $66.7 \%$ & $43.1 \%-84.5 \%$ \\
\hline
\end{tabular}




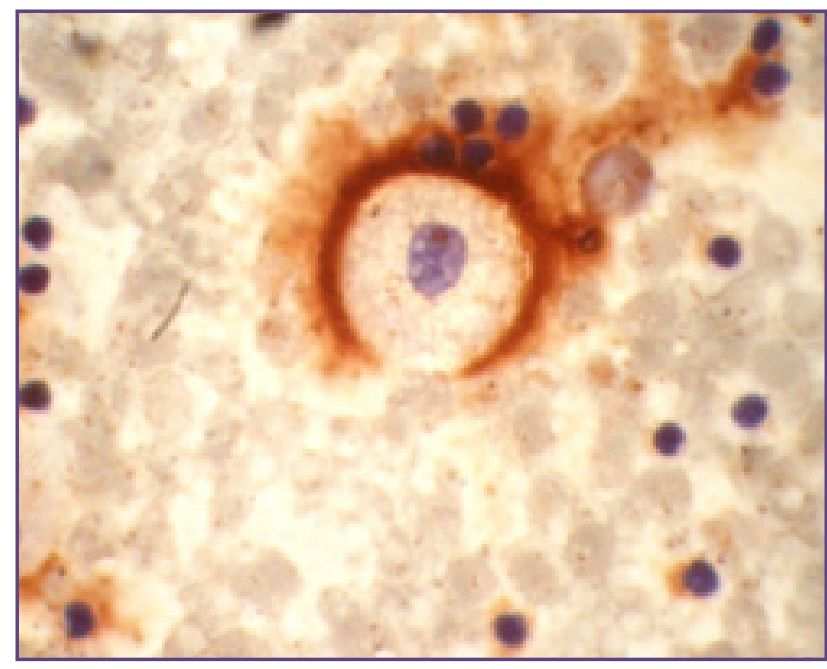

Fig. 1: Membranous staining seen in reactive mesothelial cells.(HBME1,ICC X400).

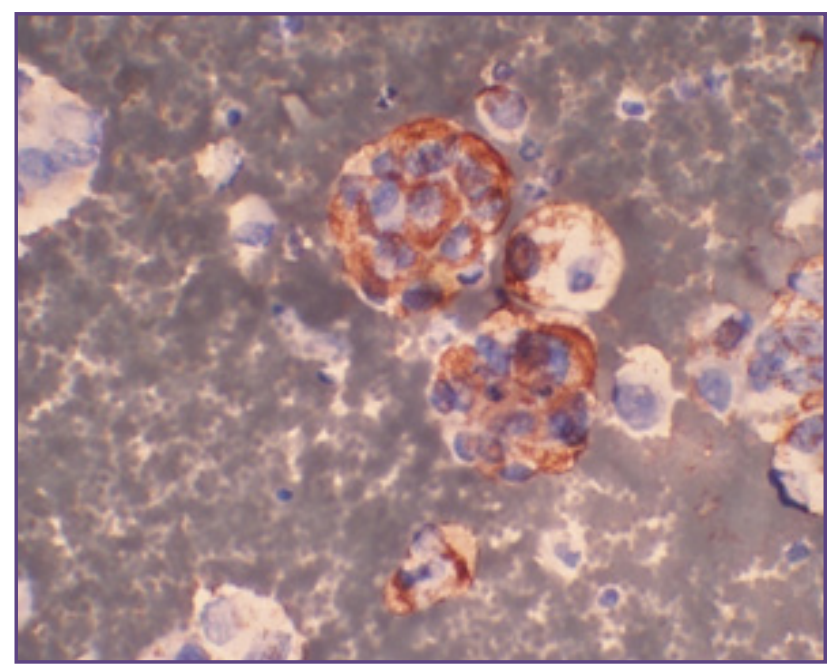

Fig. 3: Cytoplasmic staining seen in metastatic adenocarcinoma Cells..(HBME1,ICC X400).

vacuole. Adenocarcinoma being the most common malignancy in serous cavities, the differntiation between two is mandatory.HBME1 is monoclonal antibody which reacts with unknown antigen presents on mesothelial cells.Immunocytochemistry with HBME1 can be used to differentiate between reactive mesothelial cells and malignant cells, the reactive mesothelial cells show membranous pattern of staining while adenocarcinoma cells show cytoplasmic staining. ${ }^{[7-9]}$

In our study out of 18 benign cases ,14 (77.8\%) were positive for HBME1 while $4(22 \%)$ cases were negative .Out 14 positive cases 12 cases showed membranous positivity and 2 cases showed cytoplasmic positivity. These findings are in correlation to Ascoli et al ${ }^{[10]}$ study which in which

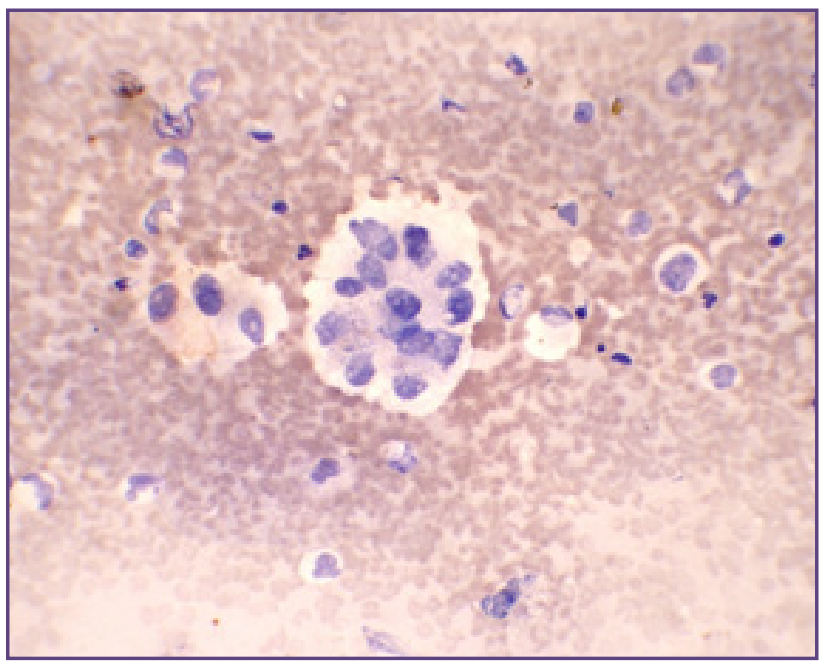

Fig. 2: Negative staining in adenocarcinoma cells. (HBME1,ICC X400).

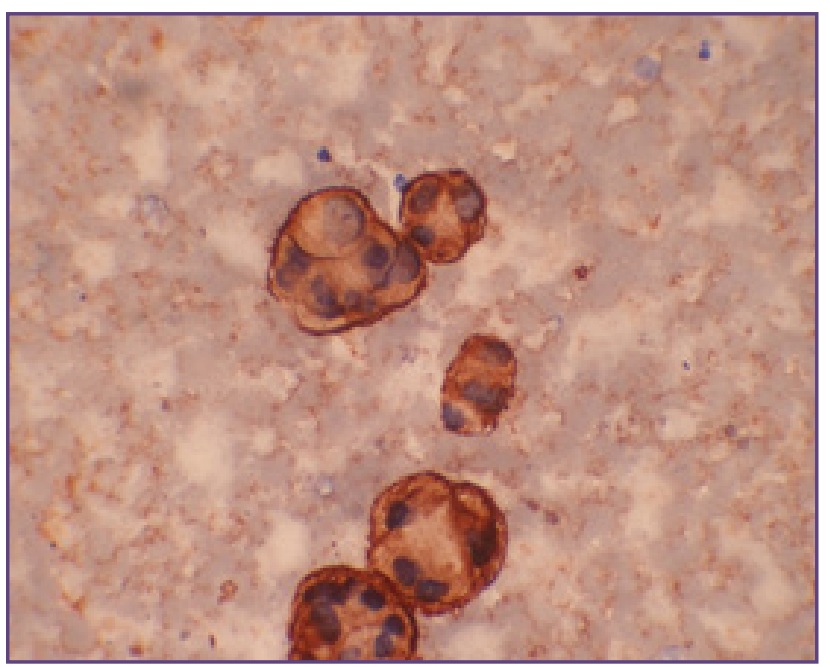

Fig. 4: Combined staining seen in metastatic adenocarcinoma Cells..(HBME1,ICC X400).

predominant pattern of staining in reactive mesothelial cells was membranous while few cells showed cytoplasmic positivity..Study conducted by Rehmani et al. ${ }^{[1]}$ showed $100 \%$ membranous positivity in reactive mesothelial cells while none of the cells showed combined positivity. These findings suggest predominant staining pattern for benign mesothelial cells is membranous.

Out of 42 malignant cases 28 (66.7\%) were negative for HBME1 while 14 (33.3\%) cases were positive .In Ascoli et al ${ }^{[10]}$ study $24 \%$ malignant cases showed positive staining with HBME1.

The most common malignancy was adenocarcinoma, total 36 cases,22(61.1\%) cases were negative for HBME1 staining while $14(38.9 \%)$ cases showed positivity. Out 
of positive cases $8(57.1 \%)$ cases showed cytoplasmic positivity and 4 (28.6\%)cases showed membranous positivity. Only 2 cases (14.3\%)cases showed membranous positivity ,both were ovarian adenocarcinoma.

Thus in adenocarcinoma predominant staining pattern was cytoplasmic followed by combined .Ovarian tumor cells showed membranous positivity. In Ascoli et al ${ }^{[10]}$ ovarian adenocarcinoma (83\%) showed membranous pattern while other carcinomas showed cytoplasmic immunoreactivity.

Positive membranous staining in Ovarian adenocarcinoma can be explained on the basis of origin of tumor. Ovarian adenocarcinoma originate from germline epithelium of ovary which is origin of mesothelial cells. ${ }^{[12]}$ Utility of HBME1 is limited to differentiate single scattered cells from ovarian adenocarcinoma and reactive mesothelial cells.

Positive predictive value and negative predictive value was $50 \%$ and $87.5 \%$ respectively .[TableVI ] The sensitivity and specificity of immunomarker was $77.8 \%$ and $66.7 \%$ respectively. According to Politi et al ${ }^{[13]}$ the sensitivity and specificity for HBME-1 was $98 \%$ and $71 \%$ respectively.

\section{Conclusion}

Staining pattern with HBME1 is useful in differentiating reactive mesothelial cells and adenocarcinoma cells .In case of ovarian adenocarcinoma the utility of HBME1 is limited due to similar immunoreactivity as reactive mesothelial cells .

\section{References}

1. Lozano MD, Panizo A, Toledo GR, Sola JJ, Pardo-Minda J. Immunocytochemistry in the differential diagnosis of serous effusions. A comparative evaluation of eight monoclonal antibodies in Papanicolaou stained smears. Cancer Cytopathol 2001;93:68-72.

2. Fetsch PA, Abati A. Immunocytochemistry in effusion cytology. Cancer Cytopathol 2001;93:293-308.

3. Sayed DM, el-Attar MM, Hussein AA. Evaluation of flow cytometric immunophenotyping and DNA analysis for detection of malignant cells in serosal cavity fluids. Diagn Cytopathol 2009;37:498-504.

4. Krausz T, Barker F. Metastatic disease and lymphomas. In: Gray W, McKee GT, editors. Diagnostic Cytopathology. 2nd ed. London: Churchill- Livingstone; 2003. p. 135-200.

5. Murugan P, Siddaraju N, Habeebullah S, Basu D. Immunohistochemical distinction between mesothelial and adenocarcinoma cells in serous effusions: A combination panel-based approach with a brief review of the literature. Indian J Pathol Microbiol 2009;52:175-81.

6. Henzi T, Blum WV, Pfefferli M, Kawecki TJ, Salicio V, Schwaller B. SV40-induced expression of calretinin protects mesothelial cells from asbestos cytotoxicity and may be a key factor contributing to mesothelioma pathogenesis. Am J Pathol 2009;174:2324-36.

7. Wu GP, Zhang SS, Fang CQ, Liu SL, Wang EH. Immunocytochemical panel for distinguishing carcinoma cells from reactive mesothelial cells in pleural effusions. Cytopathology 2008;19:212-7.

8. MocanuL, Cîmpean AM, Raica M. Value of antimesothelioma HBME-1 in the diagnosis of inflammatory and malignant pleural effusions. Rom J Morphol Embryol 2006;47:351-5.

9. Marchevsky AM, Wick MR. Evidence-based guidelines for the utilization of immunostains in diagnostic pathology: Pulmonary adenocarcinoma versus mesothelioma. Appl Immunohistochem Mol Morphol 2007;15:140-4.

10. Ascoli V, Carnovale-Scalzo C, Taccogna S, Nardi F. Utility of HBME- 1 immunostaining in serous effusions. Cytopathology 1997;8:328-35.

11. Rehmani A, Dehgani MZ, Afshar NM, Heidarian H, Tahririan R. HBME-1 immunostaining in reactive mesothelial versus metastatic adenocarcinoma cells in serous fluid. Indian $\mathrm{J}$ Pathol Microbiol. 2011;54:460-3.

12. Szczepulska-Wójcik E, Langfort R, Roszkowski-Sliz K. A comparative evaluation of immunohistochemical markers for the differential diagnosis between malignant mesothelioma, non-small cell carcinoma involving the pleura, and benign reactive mesothelial cell proliferation. Pnumonol Alergol Pol 2007;75:57-69.

13. Politi E, Kandaraki C, Apostolopoulou C, Kyritsi T, Koutselini H. Immunocytochemical panel for distinguishing between carcinoma and reactive mesothelial cells in body cavity fluids. Diagn Cytopathol 2005;32:151-5.

*Corresponding author:

Dr Rupali Bavikar, C 505, Siciliaa, BT Kawade Road, Pune 01, India

Phone: +91 8149367249

Email: rupalibavikar@yahoo.co.uk

Funding: Dr. D. Y. Patil Vidyapeeth, Pune

Financial or other Competing Interests: None. 